\title{
ADVANCES IN ORBIT DRIFT CORRECTION IN THE ADVANCED PHOTON SOURCE STORAGE RING
}

\author{
L. Emery M. Borland \\ Advanced Photon Source, Argonne National Laboratory \\ 9700 South Cass Avenue, Argonne, Illinois 60439 USA
}

CONF-970503--65

\section{Abstract}

The Advanced Photon Source storage ring is required to provide X-ray beams of high positional stability, specified as $17 \mu \mathrm{m} \mathrm{rms}$ in the horizontal plane and $4.4 \mu \mathrm{m} \mathrm{rms}$ in the vertical plane. We report on the difficult task of stabilizing the slow drift component of the orbit motion down to a few microns rms using workstation-based orbit correction. There are two aspects to consider separately-the correction algorithm and the configuration of the beam position monitors (BPMs) and correctors. Three notable features of the correction algorithm are: low-pass digital filtering of BPM readbacks; "despiking" of the filtered orbit to desensitize the orbit correction to spurious BPM readbacks without having to change the correction matrix; and BPM intensity-dependent offset compensation. The BPM/corrector configuration includes all of the working BPMs but only a small set of correctors distributed around the ring. Thus only those orbit modes that are most likely to be representative of real beam drift are handled by the correction algorithm.

\section{INTRODLCTION}

The mathematics of orbit correction is covered extensively in the literature. In this paper, we discuss the application of these well-known ideas in a realistic situation with non-ideal and possibly malfunctioning BPMs. We also discuss practical issues such as how many BPMs and correctors to use, and mention potential pirfalls.

The main problems we encountered in orbit drift correction are the dependence of $r i$ BPM electrical offset on bunch train intensity and on bunch uniformity within the detected bunch train. The range of offset variation for individual BPMs is actually several times the stability specification (see below), requiring use of compensation schemes to estimate the true orbit from the BPM system data. The intensity-dependent offset variation is largely reproducible and is regarded as a systematic error that san be taken into account. The bunch train uniformity dependence of offset is more difficult to quantify and is considered an error that is randomized at every fill. In addition. we developed a way to handle intermittent bad BPM data without interrupting correction and having to remove the $B P M$ from the correction matrix.

The positron beam in the Advanced Photon Source (APS) storage ring must be stable to $5 \%$ of its size. The specified limit on rms orbit motion at the insertion device (ID) source points and the actual uncorrected orbit motions are given in Table 1 . Slow motion is seen by the user as an unwanted beam steering. while the fast motion is seen as an unwanted emittance growth.
Table 1: Orbit Motion Specification and Measurements in Various Frequency Ranges

\begin{tabular}{|l|c|c|}
\hline & \multicolumn{1}{|c|}{$\begin{array}{c}\mathrm{y} \\
\beta_{\mathrm{x}}=15 \mathrm{~m}\end{array}$} & $\begin{array}{c}\mathrm{y} \\
\beta_{\mathrm{y}}=10 \mathrm{~m}\end{array}$ \\
\hline \hline $\begin{array}{l}\text { stability } \\
\text { specification }\end{array}$ & $17 \mu \mathrm{m}$ & $4.5 \mu \mathrm{m}$ \\
\hline $\begin{array}{l}\text { slow motion } \\
\left(10^{-4}-10^{-2} \mathrm{~Hz}\right)\end{array}$ & $18 \mu \mathrm{m}$ & $5 \mu \mathrm{m}$ \\
\hline $\begin{array}{l}\text { slow motion after } \\
\text { correction }\end{array}$ & $2 \mu \mathrm{m}$ & $2 \mu \mathrm{m}$ \\
\hline $\begin{array}{l}\text { "fast" motion } \\
\left(10^{-2}-50 \mathrm{~Hz}\right)\end{array}$ & $17 \mu \mathrm{m}$ & $6 \mu \mathrm{m}$ \\
\hline
\end{tabular}

The uncorrected orbit drift rms amplitude has not been uniform in all running periods, but it has reached values as high as those listed in Table 1. As the data show, the workstation-based orbit correction handles the slow motion quite effectively, but can do nothing about the fast motion. A real-time orbit feedback system has been designed and tested to reduce the orbit motion up to $50 \mathrm{~Hz}[1,2]$. The latter system should be ready for operations soon. However, since it may encounter difficulties at low frequencies due to some of the problems discussed below, it is anticipated that the workstation-based system will continue to be used to correct orbit drift.

The correction system is implemented on a Unix workstation with Tcl/Tk interfaces and simple SDDS-compliant toolkit programs that communicate with our EPICS control system. The flexibility of this system, which has been described elsewhere [3], has allowed us rapid turnaround in orbit correction implementation changes.

\section{GENERAL CONSIDERATIONS}

There are a number of issues that are common to any orbit correction algorithm. Orbit correction consists of reading BPM data from the control system and processing this data so that it reflects as much as possible the real orbit with the appropriate frequency components. This involves correcting systematic errors and, if needed, averaging over time or filtering. This set of values is a vector that is multiplied by a correction matrix (in our case a corrector-BPM response matrix that is inverted using SVD). The result is a vector of corrector strength changes that is then applied, typically after multiplication by a dimensionless "gain" factor that is between 0 and 1 .

Generally, the algorithm waits an interval after making a change before repeating the whole operation. It is 


\section{DISCLAMMER}

Portions of this document may be illegible in electronic image products. Images are produced from the best available original document. 


\section{DISCLAMER}

This report was prepared as an account of work sponsored by an agency of the United States Government. Neither the United States Government nor any agency thereof, nor any of their employees, makes any warranty, express or implied, or assumes any legal liability or responsibility for the accuracy, completeness, or usefulness of any information, apparatus, product, or process disclosed, or represents that its use would not infringe privately owned rights. Reference herein to any specific commercial product, process, or service by trade name, trademark, manufacturer, or otherwise does not necessarily constitute or imply its endorsement, recommendation, or favoring by the United States Government or any agency thereof. The views and opinions of authors expressed herein do not necessarily state or reflect those of the United States Government or any agency thereof. 
desirable to make orbit corrections as frequently as possible so that any orbit change can be acted upon as soon as possible. However, the frequency of correction is always limited by practical considerations. In our case, it is limited by the response time of the correctors in reaching their full setpoint value, the need to wait sufficient time for the BPMs to settle to new readbacks. the need to wait for averaging of BPM readbacks. and the response time of the control system. A conservative wait time of 5 seconds guarantees that the new orbit has settled.

As mentioned above, the gain is the fraction of the correction to be applied at every iteration. Given that the correction matrix (whether from a model or experiment) is never known exactly and that the BPMs are not perfectly precise, it is necessary to use a gain lower than 1 for the orbit to converge to the target orbit. Of course, the speed of the correction is reduced by the factor of the gain. We typically use gain factors of 0.4 .

\section{ORBIT DATA PREPARATION}

Proper preparation of the orbit data proved critical to the success of our system. Some of the algorithms we used are new or specific to APS systems.

\subsection{Low-Pass Filtering}

Low-pass filtering provides sampled BPM data in the frequency band of interest. If the sampled BPM data contain frequencies above half the sampling frequency, the orbit correction will be effectively injecting noise into the lower frequency band. In our system a 2048-turn running average of the orbit position is produced by low-level digital hardware and is made available to local CPUs at a 10 $\mathrm{Hz}$ rate. This data is processed by the local CPUs through a one-pole digital filter of a time constant of $10 \mathrm{~s}$. Although the time constant was not optimized in a systematic way, it was made consistent with the correction interval. Its value was selected initially to provide a readback with an rms variation much less that the orbit drift amplitude that we wanted to eliminate

\subsection{Despiking}

Under-determined orbit correction (i.e.. orbit correction employing fewer singular values than BPMs) effectively "fits" a free betatron oscillation through the many BPMs between correctors. This firting does more harm than good when an individual BPII suffers a sudden offset shift while no real orbit drift occurred. A conventional orbit correction algorithm will generate an unwanted orbit bump of the opposite sign to the offset change and will minimize the rms of the apparent orbit error in the area. Other linear methods (e.g., best corrector or bump methods) also intrinsically do betatron oscillation fitting and hence exhibit the same behavior.

Since sudden offset shifts are a problem with the APS BPMs, a "despiking" filter is applied to the set of BPM data to smooth out obviously bad data. This filter uses information trom neighboring BPMls to test the validity of the data from each BPM. If the reading for any BPM differs from the average of its $C$ nearest neighbors by more than a chosen threshold $T$, the reading is replaced by the average of its $R$ nearest neighbors. Several passes of the filter may be applied. This stage of the processing is a nonlinear operation, and distinguishes it from the conventional linear correction. The average value of the neighbors may not be the actual orbit value at the suspect BPM, but the reasonable and lower-valued guess allows the orbit correction to proceed without creating an unwanted bump.

During a user store at APS, we use a despiking threshold $\mathrm{T}$ of $20 \mu \mathrm{m}$, compare to $C=36$ nearest neighbors, replace suspect points with the average of $R=4$ nearest neighbors, and employ two passes of the filter. Figure 1 shows an example of despiking of an orbit readback that has obviously bad data.

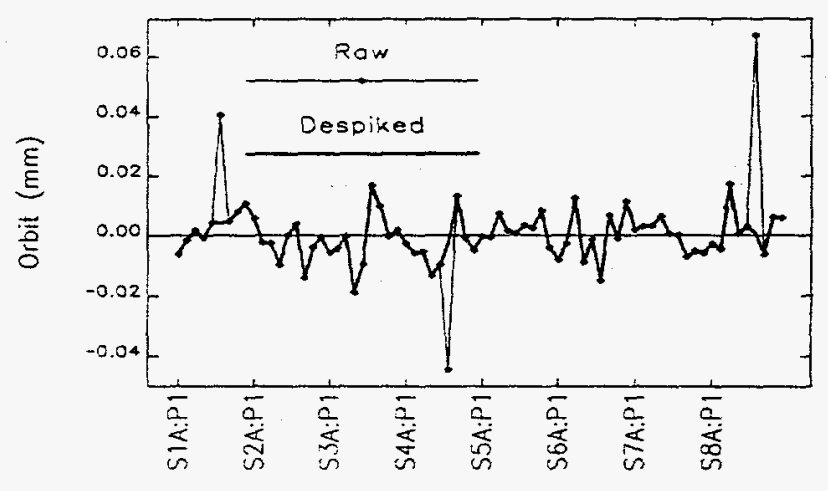

Figure 1: Raw and despiked orbit data for part of the ring.

Another approach to bad BPM data would be to ignore it altogether. This is impractical, as it requires a time-consuming recomputation of the inverse response matrix. It is very efficient to replace the suspect BPM value with another value rather than simply ignoring the BPM in the correction.

\subsection{Offset Compensation}

In general all BPM systems suffer to some degree from electrical offset variation with bunch train intensity and bunch pattern. For a fixed-bunch pattern, the offset variation as the current decays is in principle reproducible. A measurement of the intensity-dependent offset for all BPMs can be performed and the resulting data used to adjust the BPM readbacks during a user store. The measurement consists of scraping down the beam over a period of about 10 minutes while the orbit correction is running, while simultaneously reading BPM data at short intervals. Typically, we do many such experiments and choose the one that gives the cleanest result. The variation for each BPM is fit with a quadratic as a function on intensity and used to create a look-up table for offset adjustments.

Figure 2 shows offset data for some BPMs from the look-up table. The sum signal of a selected BPM is used as a measure in intensity. At the beginning of a store, the intensity is maximum, and the offset adjustment is necessarily zero for all BPMs. 


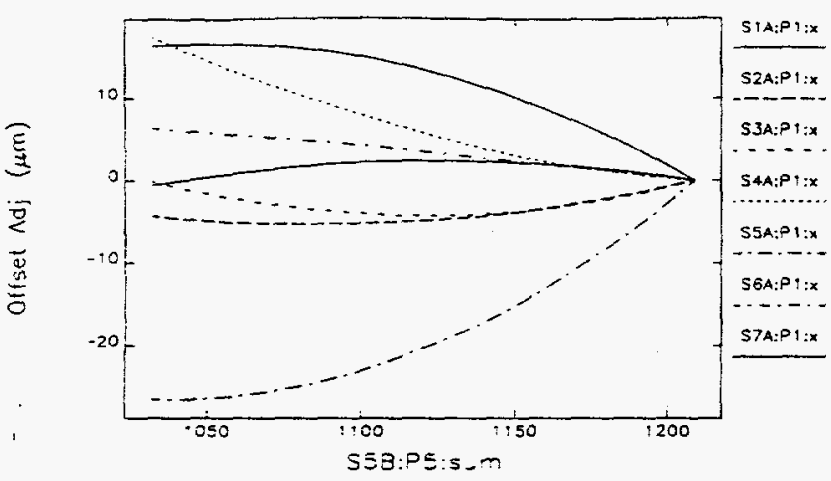

Figure 2: Fitted offset data for some BPMs.

\section{BPM/CORRECTOR CONFIGLRATION}

\subsection{Global Orbit Correction}

The correction configuration refers to the set of BPMs and correctors used in the correction matrix. In addition, the number of singular values used in the SVD inversion procedure should be considered part of the configuration. We have optimized our orbit drift correction for the case of several weak sources.

In such a case, we expect a smooth-looking orbit composed of a few harmonics near the tune value. While the latter is true in the case of larger individual sources, an orbit kink is present at such a source. This makes the orbit hard to correct unless one of the correctors of the configuration happens to be very near the source.

The correction configuration is comprised of all working BPMs and relatively few correctors. We use as many BPMs as possible in order to reduce the importance of any individual BPM readback. This also increases the effectiveness of the despiking filter mentioned above.

Since we are correcting many small, distributed sources, we use relatively few correctors distributed around the ring: two correctors for each sector that is equipped with an ID, and one in sectors with no IDs. We kept all the singular values in the matrix in order to have the selected correctors acting independently and as locally as possible. The two correctors in each ID sector are particularly good at compensating the $\sim 20 \mu \mathrm{m}$ global orbit perturbation that occurs from opening or closing the ID. Here, the orbit very close to the ID is not maintained completely. However the local orbit should have been locally steered earlier with the gaps closed. Thus repeatedly closing the ID gap with global orbit correction running should return the X-ray beam to the previously steered position.

Using many more BPMs than correctors and the implied fitting of the despiking orbit has the additional effect of controlling the $\mathrm{X}$-ray beam positions better that the noise level of the $f$ BPMs in the frequency range of the orbit drift correction [4].

The nonreproducibility of the uniformity of the bunch train contributes to the fill-to-fill orbit variability. To alleviate the problem one can reinject bunch trains until one of sufficient uniformity is obtained. In addition, one can restore the corrector setpoints at the end of the previous fill and run a global correction on the new fill. The global correction is expected to correct any real orbit drift that may occur in the time between the fills and de-emphasize the offset change of individual BPMs. The effectiveness of this procedure is evaluated using bending magnet source $\mathrm{X}$-ray BPMs readbacks over several fills.

A local source of orbit drift will cause the nearby correctors active in the global correction to move in a highly correlated manner. Searching for correlated correctors is expected to aid in locating unknown sources.

\subsection{Local Steering}

Local orbit bumps are occasionally applied to adjust the position of the X-ray beam at the end of an X-ray beamline. In practice, bump coefficients derived from a lattice model or measured response matrix do not produce an exactly matched bump, particularly in a machine with strong nonlinearities. Our solution is to create an orbit correction configuration using two BPMs on either side of the source point and 40 others distributed evenly around the ring. Two pairs of correctors on each side of the source point provide steering. The resulting $4 \times 42$ correction matrix is very over-constrained. While the first iteration of the local correction will be unmatched to whatever degree the bump coefficients are inexact, subsequent iterations will correct for this.

\section{ACKNOWLEDGMENTS}

We thank F. Lenkszus and K. Evans for the programming work they contributed to orbit correction. We thank J. Galayda for several insights into orbit correction.

This work was supported by the U.S. Department of Energy, Office of Basic Energy Sciences, under Contract No. W-31-109-ENG-38

\section{REFERENCES}

[1] J. Carwardine, K. Evans, "Evaluation of the Global Orbit Correction Algorithm for the APS Real-Time Orbit Feedback System," these proceedings.

[2] J. Carwardine, G. Decker, K. Evans, A. Hillman, F. Lenkszus, R. Merl, A. Pietryla, "Commissioning and Performance of the APS Real-Time Orbit Feedback System," these proceedings.

[3] M. Borland, "Applications Toolkit for Accelerator Control and Analysis," these proceedings.

[4] G. Decker, "Performance of the APS Storage Ring," these proceedings. 